CONFORMAL GEOMETRY AND DYNAMICS

An Electronic Journal of the American Mathematical Society

Volume 11, Pages 107-127 (July 26, 2007)

S 1088-4173(07)00163-4

\title{
NORMAL COVERINGS OF HYPERELLIPTIC REAL ALGEBRAIC CURVES
}

\author{
E. BUJALANCE, F. J. CIRRE, AND J. M. GAMBOA
}

\begin{abstract}
We consider normal (possibly) branched, finite-sheeted coverings $\pi: X \rightarrow X^{\prime}$ between hyperelliptic real algebraic curves. We are interested in the topology of such coverings and also in describing them in terms of algebraic equations. In this article we completely solve these two problems in case $X$ has the maximum number of ovals within its genus. We first analyze the topological features and ramification data of such coverings. For each isomorphism class we then describe a representative, with defining polynomial equations for $X$ and for $X^{\prime}$, formulae for generators of the covering transformation group, and a rational formula for the covering $\pi: X \rightarrow X^{\prime}$.
\end{abstract}

\section{INTRODUCTION}

There exists an extensive literature dealing with finite-sheeted, normal branched coverings $\pi: S \rightarrow S^{\prime}$ where $S$ and $S^{\prime}$ are compact Riemann surfaces of genus at least two, and $S^{\prime}$ is hyperelliptic. Since the covering is normal, we have a finite group $G$ of covering transformations, and $S^{\prime}=S / G$.

The study of these types of coverings started with the case in which $S$ is also hyperelliptic and $\pi: S \rightarrow S^{\prime}$ is unbranched. Maclachlan [17] and Farkas [9] independently proved that in this case $G$ is either the cyclic group $C_{2}$ of order two or the non-cyclic group $D_{2}$ of order four. The tools used in the two papers are quite different; Maclachlan uses Fuchsian groups, while Farkas considers Weierstrass points. With $S^{\prime}$ fixed and up to analytic equivalence of the domain $S$, the number of such coverings has been calculated, as a function of the genus of $S^{\prime}$ in both cases; by Farkas [9], if $G=C_{2}$, and by Kato in an unpublished paper and recorded by Horiuchi [15], if $G=D_{2}$.

The above results have been generalized in two directions, each direction eliminating one of the two hypotheses.

First, keeping the assumption that $\pi: S \rightarrow S^{\prime}$ is unbranched, Maclachlan 17 showed that $S$ need not be hyperelliptic, even with $G=C_{2}$. The same result was proved by Farkas [9]. Afterwards Bujalance [4, see also Farkas [10, proved that $S$ is $p$-hyperelliptic for some integer $p$ with $p \geq 2$, and calculated the number of such

Received by the editors February 6, 2007.

2000 Mathematics Subject Classification. Primary 30F50; Secondary 14P25, 30F35.

Key words and phrases. Normal coverings, hyperelliptic real algebraic curves, combinatorial methods.

The research of the first two authors was partially supported by MEC-DGESIC (Spain) through Project MTM2005-01637.

The research of the third author was partially supported by MEC-DGESIC (Spain) through Project MTM2005-20865. 
coverings in terms of $p$. Being $p$-hyperelliptic means that $S$ occurs as a branched double cover of some surface $\bar{S}$ of genus $p$. Fuertes and González-Diez [1] proved that $\bar{S}$ is hyperelliptic and gave algebraic equations for it in terms of the ones of $S^{\prime}$. Moreover, algebraic equations of $S$ are also provided since $S$ is the fiber product of $S^{\prime}$ and $\bar{S}$ over the Riemann sphere. The same authors proved in [12] an analogous result in case the covering transformation group is $D_{2}$. On the other hand, it is worth mentioning that Accola [1] proved that for arbitrary $n$ there exists an $n$-sheeted unbranched covering $S \rightarrow S^{\prime}$ where $S$ is also hyperelliptic.

Secondly, and keeping the assumption that $S$ is hyperelliptic, Horiuchi [15] considered the more involved problem of classifying all the branched coverings $\pi: S \rightarrow S^{\prime}$. Here $G$ is no longer $C_{2}$ nor $D_{2}$ but it ranges over the set of finite subgroups of $\mathrm{SO}_{3}(\mathbb{R})$; that is, $G$ can be cyclic, dihedral, tetrahedral, octahedral or icosahedral. A major feature of Horiuchi's paper is that the coverings are described by means of algebraic equations.

We shall be studying the analogues of the above results for coverings of Klein surfaces. To be precise, we are interested in the problem of classifying, up to dianalytic equivalence in both the domain and the codomain, all the normal, branched, finite-sheeted coverings $\pi: X \rightarrow X^{\prime}$, where $X$ is a hyperelliptic, compact, Klein surface of algebraic genus at least two. Since the covering is normal, we have a finite group $G$ of covering transformations and $X^{\prime}=X / G$. It follows from a theorem of Martens [18] that the surface $X^{\prime}$ is also hyperelliptic; we provide two different proofs of this fact in Lemmas 3.1 and 4.1 .

In this paper we deal with this problem in the case where $X$ is planar; that is, its boundary has the maximum number of connected components within its algebraic genus. In addition, we just consider the non-trivial case in which $G$ does not contain the hyperelliptic involution of $X$. For simplicity, $X^{\prime}$ will be assumed to have algebraic genus at least two, although our results hold even without this condition; see Remark 4.11. Our approach is close to Horiuchi's in [15] in that we also describe coverings by means of algebraic equations. However, we fix the cover surface $X$ instead of the base surface $X^{\prime}$ as done in [15.

Branched coverings between hyperelliptic Klein surfaces have received less attention than those between Riemann surfaces; in fact, only unbranched coverings have been studied until now. It is worth mentioning that Kani [16] classified unbranched double coverings of a hyperelliptic Klein surface, extending previous results in 6 .

The paper is organized as follows. In Section 2 we recall the basic definitions and fix the notation which will be employed in the sequel. In Section 3 we study finite-sheeted, normal, branched coverings $\pi: X \rightarrow X^{\prime}$ from the combinatorial point of view. We prove that $G$ is either cyclic or dihedral, and, in Theorems 3.3 and 3.5 we obtain complete data about the topology of the base surface $X^{\prime}$ and the ramification data of the covering. Section 4 is devoted to finding an explicit algebraic description of representatives of all dianalytic equivalence classes of such coverings. This means to provide defining polynomial equations of both $X$ and $X^{\prime}$, the formulae of generators for the covering transformation group $G$, and a rational formula for the covering projection $\pi$. These are contained in Theorems 4.3 and 4.5. which are the main results of this Section.

The combinatorial and the algebraic approaches are complementary. In Proposition 4.7 we establish the correspondence between the combinatorial models in Section 3 and the algebraic models in Section 4 . 


\section{Preliminaries}

A Klein surface is a connected topological surface possibly with boundary together with an atlas whose transition functions are dianalytic, that is, either analytic or antianalytic; see [3, \$1.2]. Recall that a function is antianalytic if its composite with complex conjugation is analytic. Every compact surface can be given the structure of a Klein surface; see [3, §1.7]. For example, if $S$ is a compact Riemann surface and $\tau$ is a symmetry of $S$, that is, an antianalytic involution of $S$, then $S /\langle\tau\rangle$ is a Klein surface.

We next recall that every Klein surface arises in this way in an essentially unique way; see [3, §1.6]. Let $X$ be a compact Klein surface which is not a Riemann surface. There exists a compact Riemann surface $S$, and a symmetry $\tau$ of $S$, and a double covering $f: S \rightarrow X$ such that $f \tau=f$; thus, $S /\langle\tau\rangle$ is isomorphic to $X$ as a Klein surface. The triple $(S, \tau, f)$ is called the complex double cover of $X$; it is unique up to analytic isomorphism. The algebraic genus of $X$ is defined to be the genus of $S$.

Harnack [14] showed that the boundary of $X$ has at most $g+1$ connected components, where $g$ is the algebraic genus of $X$. It is said that $X$ is planar if its boundary has the maximum number $g+1$ of connected components.

A compact Riemann surface $S$ is said to be hyperelliptic if it is a double covering of the Riemann sphere; that is, $S$ admits an analytic involution $h$ such that the genus of the quotient $S /\langle h\rangle$ is zero. For example, it is well known that if the genus of $S$ is at most two, then $S$ is hyperelliptic. A compact Klein surface $X$ is hyperelliptic if it admits an involution $h$ such that the quotient $X /\langle h\rangle$ has algebraic genus zero. This is equivalent to its complex double cover $S$ being hyperelliptic, 6]. It is well known that if the algebraic genus of $X$ is at most 2, then $X$ is hyperelliptic.

In this article we deal with coverings $f: T \rightarrow T / \Omega$ where $T$ is a Riemann or Klein surface and $\Omega$ is a group of automorphisms of $T$. For such coverings, it makes sense to define the branching order at a branch point $q \in T / \Omega$ as the common value of the multiplicity of $f$ at each point in the fiber of $q$.

\section{Combinatorial approach}

Throughout this section, $X$ will be a hyperelliptic planar compact Klein surface of algebraic genus $g \geq 2$, and so its boundary has $g+1$ connected components. As said above, our purpose is to describe all normal branched $n$-sheeted coverings $\pi: X \rightarrow X^{\prime}$ where $X^{\prime}$ is a compact Klein surface.

The combinatorial approach to be developed in this section relies heavily on the theory of Fuchsian and non-euclidean crystallographic (NEC in short) groups. For the reader's convenience we state here the basic facts of this theory which will be used in the paper. A reference for them is [7, Chapters 0,1].

The signature $\sigma(\Delta)$ of an NEC group $\Delta$ is a collection of symbols and nonnegative integers of the form

$$
\sigma(\Delta)=\left(\gamma ; \pm ;\left[m_{1}, \ldots, m_{r}\right] ;\left\{\left(n_{11}, \ldots, n_{1 s_{1}}\right), \ldots,\left(n_{k 1}, \ldots, n_{k s_{k}}\right)\right\} .\right.
$$

The integer $\gamma$ is the topological genus of the quotient surface $U / \Delta$, where $U$ stands for the hyperbolic plane, and the sign "+" or "-" appears according to $U / \Delta$ being orientable or not. The projection $U \rightarrow U / \Delta$ has $r$ interior branch points, say $q_{1}, \ldots, q_{r}$. The integer $m_{i}$ is the branching order at $q_{i}$ of the projection $U \rightarrow U / \Delta$. The boundary of $U / \Delta$ has $k$ connected components, and $n_{j 1}, \ldots, n_{j s_{j}}$ are the 
branching orders at the $s_{j}$ branch points of $U \rightarrow U / \Delta$ lying on the $j$ th boundary component of $U / \Delta$.

The area of an NEC group $\Delta$ with the above signature is defined to be

$$
\operatorname{Area}(\Delta)=2 \pi\left(\alpha \gamma+k-2+\sum_{i=1}^{r}\left(1-\frac{1}{m_{i}}\right)+\frac{1}{2} \sum_{i=1}^{k} \sum_{j=1}^{s_{i}}\left(1-\frac{1}{n_{i j}}\right)\right),
$$

where $\alpha=2$ if $U / \Delta$ is orientable, and $\alpha=1$ otherwise. If $\Delta$ is a subgroup of finite index $\left[\Delta^{\prime}: \Delta\right]$ of another NEC group $\Delta^{\prime}$, then the Hurwitz-Riemann formula holds:

$$
\operatorname{Area}(\Delta)=\left[\Delta^{\prime}: \Delta\right] \cdot \operatorname{Area}\left(\Delta^{\prime}\right)
$$

The algebraic genus $g$ of the compact surface $U / \Delta$ is the topological genus of its complex double cover. It satisfies the equality $g=2 \gamma+k-1$ if $U / \Delta$ is orientable and $g=\gamma+k-1$ otherwise. If the number of boundary components attains its maximum value $k=g+1$, then $\gamma=0$ and the quotient $U / \Delta$ is a sphere with holes. If the number of boundary components is $k=g$, then $\gamma=1$ and the quotient $U / \Delta$ is a projective plane with holes.

On the other hand, the signature $\sigma(\Delta)$ provides a presentation of $\Delta$ as an abstract group. It has generators $x_{1}, \ldots, x_{r}$ (elliptic elements), $c_{10}, \ldots, c_{1 s_{1}}, \ldots, c_{k 0}$, $\ldots, c_{k s_{k}}$ (reflections), $e_{1}, \ldots, e_{k}$ (orientation preserving elements), $a_{1}, b_{1}, \ldots, a_{\gamma}, b_{\gamma}$ (hyperbolic elements in case "+"), $d_{1}, \ldots, d_{\gamma}$ (glide reflections in case "-"), and defining relations

$$
\begin{aligned}
& \text { - } x_{i}^{m_{i}}=1 \text {, for } 1 \leq i \leq r \\
& \text { - } e_{i} c_{i s_{i}}=c_{i 0} e_{i} \text { and } c_{i j-1}^{2}=c_{i j}^{2}=\left(c_{i j-1} c_{i j}\right)^{n_{i j}}=1 \text { for } 1 \leq i \leq k ; 1 \leq j \leq s_{i} \text {; } \\
& \text { - } x_{1} \cdots x_{r} e_{1} \cdots e_{k} a_{1} b_{1} a_{1}^{-1} b_{1}^{-1} \cdots a_{\gamma} b_{\gamma} a_{\gamma}^{-1} b_{\gamma}^{-1}=1 \text { (in case "+"); } \\
& \text { - } x_{1} \cdots x_{r} e_{1} \cdots e_{k} d_{1}^{2} \cdots d_{\gamma}^{2}=1 \text { (in case "-"). }
\end{aligned}
$$

A set of generators, as the above, is called a canonical set of generators of $\Delta$. The integers $m_{1}, \ldots, m_{r}$ are the proper periods of $\sigma(\Delta)$, and $\left(n_{j 1}, \ldots, n_{j s_{j}}\right)$ are the link periods of the $j$ th period cycle of $\sigma(\Delta)$. When $k=1$, the first subindex of $c_{i j}$ and $n_{i j}$ is omitted. A surface NEC group is an NEC group with no non-trivial orientation preserving elements of finite order. In such a case, we write its signature as $(\gamma ; \pm ;[-] ;\{(-), . . ., .,(-)\})$.

By the Riemann uniformization theorem, a compact Klein surface $X$ of algebraic genus $g \geq 2$ can be written as $X=U / \Gamma$, where $\Gamma$ is a surface NEC group. If $X$ is hyperelliptic and planar, then it follows by [6] that there exists an NEC group $\Gamma_{h}$ containing $\Gamma$ as a subgroup of index 2 whose signature is

$$
\sigma\left(\Gamma_{h}\right)=\left(0 ;+;[-] ;\left\{\left(2,{ }^{2 g+2}, 2\right)\right\}\right) .
$$

The quotient $\Gamma_{h} / \Gamma$ is generated by the hyperelliptic involution $h$ of $X$.

The covering transformation group $G$ of the $n$-sheeted covering $\pi: X \rightarrow X^{\prime}$ is a group of automorphisms of $X$, and so we may write $G=\Gamma^{\prime} / \Gamma$ for some NEC group $\Gamma^{\prime}$ containing $\Gamma$ as a normal subgroup of index $n$. Note that $X^{\prime}=X / G=U / \Gamma^{\prime}$ and so $\Gamma^{\prime}$ uniformizes $X^{\prime}$ although $\Gamma^{\prime}$ is not necessarily a surface group.

Assume for a while that $\Gamma_{h} \subseteq \Gamma^{\prime}$. Then the group $\Gamma_{h} / \Gamma$ is contained in $\Gamma^{\prime} / \Gamma=G$. Thus, $X /\langle h\rangle$ covers $X / G=X^{\prime}$; since $X /\langle h\rangle$ has algebraic genus zero and nonempty boundary, the same happens to $X^{\prime}$; that is, $X^{\prime}$ is a closed disc. Since we are interested in genus bigger than one, we will assume from now on that $\Gamma_{h} \not \subset \Gamma^{\prime}$; that is, $G$ does not contain the hyperelliptic involution of $X$. 
Since $\Gamma$ is a normal subgroup of $\Gamma^{\prime}$ and $h$ is a central element in the automorphism group of $X$, it follows readily that the product $\Gamma^{\prime} \Gamma_{h}$ is a group. We will denote it by $\Gamma_{h}^{\prime}$. Notice that $\Gamma$ is a normal subgroup of $\Gamma_{h}^{\prime}$ since it is normal in both $\Gamma^{\prime}$ and $\Gamma_{h}$.

3.1. Lemma. With the above notation, the following holds:

(i) The group $\Gamma_{h}^{\prime} / \Gamma_{h}$ is isomorphic to the covering transformation group $G$.

(ii) The group $\Gamma_{h}^{\prime}$ contains $\Gamma^{\prime}$ as a subgroup of index 2, and the quotient surface $U / \Gamma_{h}^{\prime}$ has algebraic genus 0 . In particular, the surface $X^{\prime}=U / \Gamma^{\prime}$ is hyperelliptic.

(iii) The unique epimorphism $\eta^{\prime}: \Gamma_{h}^{\prime} \rightarrow C_{2}$ with $\operatorname{ker} \eta^{\prime}=\Gamma^{\prime}$ extends the unique epimorphism $\eta: \Gamma_{h} \rightarrow C_{2}$ with ker $\eta=\Gamma$.

Proof. Since $\Gamma \subseteq \Gamma^{\prime} \cap \Gamma_{h} \varsubsetneqq \Gamma_{h}$ and $\left[\Gamma_{h}: \Gamma\right]=2$, we have $\Gamma^{\prime} \cap \Gamma_{h}=\Gamma$. Thus,

$$
\frac{\Gamma_{h}^{\prime}}{\Gamma_{h}}=\frac{\Gamma^{\prime} \Gamma_{h}}{\Gamma_{h}} \simeq \frac{\Gamma^{\prime}}{\Gamma^{\prime} \cap \Gamma_{h}}=\frac{\Gamma^{\prime}}{\Gamma} \simeq G,
$$

which proves (i).

For claims in (ii), let us write $\Gamma_{h}=\Gamma \cup \Gamma u$, with $u \notin \Gamma$. Multiplying by $\Gamma^{\prime}$ yields $\Gamma_{h}^{\prime}=\Gamma^{\prime} \Gamma_{h}=\Gamma^{\prime} \cup \Gamma^{\prime} u$, which proves the first part. As to the second, we have a covering of Riemann surfaces $U / \Gamma_{h}^{+} \rightarrow U /\left(\Gamma_{h}^{\prime}\right)^{+}$, where $\Gamma_{h}^{+}$and $\left(\Gamma_{h}^{\prime}\right)^{+}$are the subgroups of orientation preserving elements of $\Gamma_{h}$ and $\Gamma_{h}^{\prime}$, respectively. Since $U / \Gamma_{h}^{+}$has genus zero and $U /\left(\Gamma_{h}^{\prime}\right)^{+}$also has genus zero, this means that the Klein surface $U / \Gamma_{h}^{\prime}$ has algebraic genus zero. In particular, since $U / \Gamma_{h}^{\prime}=X^{\prime} /\left\langle\Gamma^{\prime} u\right\rangle$ and $\Gamma^{\prime} u$ is an involution, we conclude that $X^{\prime}=U / \Gamma^{\prime}$ is hyperelliptic.

Finally, let us write again $\Gamma_{h}=\Gamma \cup \Gamma u$, with $u \notin \Gamma$. Let $x$ be an element in $\Gamma_{h}$. If $\eta(x)=0$, then $x \in \operatorname{ker} \eta=\Gamma \subsetneq \Gamma^{\prime}=\operatorname{ker} \eta^{\prime}$ and so $\eta^{\prime}(x)=0$. If $\eta(x)=1$, then $x \notin \Gamma$ and so $x \notin \Gamma^{\prime}$. Indeed, if $x \in \Gamma^{\prime}$, then we would have $\Gamma \cup \Gamma x \subseteq \Gamma^{\prime}$, which is impossible since $\Gamma \cup \Gamma x=\Gamma \cup \Gamma u=\Gamma_{h}$ and we are assuming that $\Gamma_{h} \not \subset \Gamma^{\prime}$.

The covering transformation group $G$ is a group of automorphisms of the genus $g$ hyperelliptic planar Klein surface $X$ which does not contain its hyperelliptic involution. Groups of automorphisms of such surfaces have been studied in [7. Chapter 6]. This leads us to the following proposition, where we let $C_{n}$ denote an abstract cyclic group of order $n$ and $D_{m}$ denote an abstract dihedral group of order $2 m$. We keep this notation all throughout the article.

3.2. Proposition. Let $n$ denote the order of the covering transformation group $G$. Then $G$ is either $C_{n}$ or $D_{n / 2}$ and $n$ divides $2 g+2$. If $n>2$, then the signature of the $N E C$ group $\Gamma_{h}^{\prime}$ is

$$
\sigma\left(\Gamma_{h}^{\prime}\right)=\left\{\begin{array}{lr}
(0 ;+;[n] ;\{(2, . t ., 2)\}) & \text { if } G \text { is cyclic, } \\
(0 ;+;[-] ;\{(2, .+.2,2, n / 2)\}) & \text { if } G \text { is dihedral, }
\end{array}\right.
$$

where $t=2(g+1) / n$. If $n=2$, then $\sigma\left(\Gamma_{h}^{\prime}\right)$ is either

$$
(0 ;+;[2] ;\{(2, g+., 2)\}) \text { or }(0 ;+;[-] ;\{(2, g+3,2)\}) \text {, }
$$

and both signatures occur for different actions of $G$.

Proof. The quotient group $\Gamma_{h}^{\prime} / \Gamma$ is a group of automorphisms of $X=U / \Gamma$ of even order $2 n$, which contains the hyperellipticity automorphism group $\Gamma_{h} / \Gamma$ of $X$. Thus, by [7, Thm. 6.3.1] and its proof, the group $\Gamma_{h}^{\prime} / \Gamma$ is either $C_{n} \times C_{2}$ or $D_{n / 2} \times C_{2}$, 
where the second factor $C_{2}$ stands for the hyperellipticity automorphism group. As $G=\Gamma^{\prime} / \Gamma$ is an index 2 subgroup in $\Gamma_{h}^{\prime} / \Gamma$ which does not contain the hyperelliptic involution, then $G$ is either $C_{n}$ or $D_{n / 2}$. Also in [7, Thm. 6.3.1] it is shown that the signature of $\Gamma_{h}^{\prime}$ is the one stated above. In particular, $n$ divides $2 g+2$.

Observe that double coverings have a different flavor because two distinct signatures for the group $\Gamma_{h}^{\prime}$ occur in this case. The reader will find a geometric explanation of this phenomenon in the proof of Theorem 4.9.

We are now in a position to study the coverings from a combinatorial point of view. We begin with the case $n>2$ and we consider separately the cyclic and dihedral cases.

3.3. Theorem. Let $X$ be a hyperelliptic, planar, compact, Klein surface of algebraic genus $g \geq 2$ and let $G$ be a cyclic group of order $n>2$ acting as a group of automorphisms of $X$. Assume that $G$ does not contain the hyperelliptic involution of $X$. Let $\pi: X \rightarrow X^{\prime}$ with $X^{\prime}=X / G$ be the induced normal branched $n$-sheeted covering. Then $n$ divides $g+1$ and the Klein surface $X^{\prime}$ is hyperelliptic and has $(g+1) / n$ boundary components. Moreover,

(a) if $n$ is odd, then $X^{\prime}$ is planar;

(b) if $n$ is even, then $X^{\prime}$ is either planar or a projective plane with holes, and both cases occur for different actions of $G$.

Proof. We will calculate the signature $\sigma\left(\Gamma^{\prime}\right)$ of an NEC group $\Gamma^{\prime}$ such that $X^{\prime}=$ $U / \Gamma^{\prime}$, since this signature yields the required information. Recall that the hyperellipticity of $X^{\prime}$ has been proved in Lemma 3.1. By Proposition 3.2, the signature of $\Gamma_{h}^{\prime}$ is

$$
\sigma\left(\Gamma_{h}^{\prime}\right)=(0 ;+;[n] ;\{(2, . t ., 2)\}) \text { with } t=(2 g+2) / n .
$$

Let $\eta^{\prime}: \Gamma_{h}^{\prime} \rightarrow C_{2}=\{0,1\}$ be the epimorphism with ker $\eta^{\prime}=\Gamma^{\prime}$. To calculate $\sigma\left(\Gamma^{\prime}\right)$, we determine the images under $\eta^{\prime}$ of a canonical set of generators $\left\{x_{1}^{\prime}, c_{0}^{\prime}, \ldots, c_{t}^{\prime}\right\}$ of $\Gamma_{h}^{\prime}$. A canonical set of generators $\left\{c_{0}, \ldots, c_{2 g+1}\right\}$ of $\Gamma_{h}$ consists of $c_{0}=c_{0}^{\prime}, \ldots, c_{t-1}=$ $c_{t-1}^{\prime}$ and their conjugates under non-trivial powers of $x_{1}^{\prime}$. Let us consider now the epimorphism $\eta: \Gamma_{h} \rightarrow C_{2}$ with ker $\eta=\Gamma$. It follows from [7, Prop. 2.4.3] that the images $\eta\left(c_{0}\right), \ldots, \eta\left(c_{2 g+1}\right)$ can be assumed to be $\eta\left(c_{2 i}\right)=1, \eta\left(c_{2 i+1}\right)=0$ for $0 \leq i \leq g$. Moreover, since $\eta^{\prime}$ extends $\eta$ by Lemma 3.1 and $c_{t}=x_{1}^{\prime} c_{0}^{\prime} x_{1}^{\prime-1}$ we deduce $\eta\left(c_{t}\right)=\eta\left(c_{0}\right)$. So $t$ is even; that is, $n$ divides $g+1$. Also $\eta^{\prime}\left(c_{2 i}^{\prime}\right)=1$ and $\eta^{\prime}\left(c_{2 i+1}^{\prime}\right)=0$ for $0 \leq i \leq t / 2-1$ and $\eta^{\prime}\left(c_{t}^{\prime}\right)=1$.

Moreover, according to $\eta^{\prime}\left(x_{1}^{\prime}\right)$ being 0 or 1 it follows from [7, Thms. 2.2.4, 2.3.3] and the Riemann-Hurwitz formula, that the signature of $\Gamma^{\prime}$ is either

$$
\left(0 ;+;[n, n] ;\left\{(-),{ }^{(g+1) / n},(-)\right\}\right) \text { or }(1 ;-;[n / 2] ;\{(-),(g+1) / n,(-)\}),
$$

where $n$ is even in the second signature. In both cases $X^{\prime}$ has $(g+1) / n$ boundary components, and it is a planar surface in the first case and a projective plane with holes in the second one.

3.4. Remark. The signature of $\Gamma^{\prime}$ does not only give the topological features of $X^{\prime}$ but it also contains information about the covering $\pi: X \rightarrow X^{\prime}$. (This also holds for the dihedral case, which is the subject of Theorem 3.5.) For instance, since all its period cycles are empty, $\pi$ has no branch points in the boundary of $X^{\prime}$. However, since $\sigma\left(\Gamma^{\prime}\right)$ has proper periods, $\pi$ does have ramification over the interior; over two points with branching order $n$ in the first case, and over one point with branching 
order $n / 2$ in the second. A more explicit description of the ramification can be obtained from the analysis of the coverings by means of algebraic equations done in Section 4 .

We now deal with dihedral coverings. Recall that in this case, $n$ is an even divisor of $2 g+2$.

3.5. Theorem. Let $X$ be a hyperelliptic, planar, compact, Klein surface of algebraic genus $g \geq 2$ and let $G$ be a dihedral group of order $n>2$ acting as a group of automorphisms of $X$. Assume that $G$ does not contain the hyperelliptic involution of $X$. Let $\pi: X \rightarrow X^{\prime}$ with $X^{\prime}=X / G$ be the induced normal branched $n$-sheeted covering. Then $X^{\prime}$ is a planar hyperelliptic Klein surface and the number of its boundary components is

(a) either $\frac{g+1}{n}$ or $\frac{g+1}{n}+1$ if $n$ divides $g+1$,

(b) either $\frac{g+1}{n}+\frac{1}{2}$ or $\frac{g+1}{n}+\frac{3}{2}$ otherwise.

The two values given in each case (a) or (b) do occur for different actions of $G$.

Proof. As in the proof of Theorem 3.3. we calculate the signature of an NEC group $\Gamma^{\prime}$ such that $X^{\prime}=U / \Gamma^{\prime}$. Recall that the hyperellipticity of $X^{\prime}$ has been proved in Lemma 3.1. By Proposition 3.2 the NEC group $\Gamma_{h}^{\prime}$ has the following signature:

$$
\sigma\left(\Gamma_{h}^{\prime}\right)=(0 ;+;[-] ;\{(2, \stackrel{t+2}{.}, 2, n / 2)\}) \quad \text { with } t=2(g+1) / n .
$$

Let $\left\{c_{0}^{\prime}, \ldots, c_{t+2}^{\prime}\right\}$ be a canonical set of generating reflections of $\Gamma_{h}^{\prime}$. It follows that a canonical set of generators $\left\{c_{0}, \ldots, c_{2 g+1}\right\}$ of $\Gamma_{h}$ consists of $c_{0}=c_{1}^{\prime}, \ldots, c_{t}=$ $c_{t+1}^{\prime}$, the conjugates of $\left\{c_{1}^{\prime}, \ldots, c_{t}^{\prime}\right\}$ under the orientation reversing elements of the dihedral group $\left\langle c_{0}^{\prime}, c_{t+2}^{\prime}\right\rangle$ and the conjugates of $\left\{c_{2}^{\prime}, \ldots, c_{t+1}^{\prime}\right\}$ under its non-trivial orientation preserving elements.

It follows from [7. Prop. 2.4.3] and Lemma 3.1 that for some $\alpha=0$ or 1 , we have $\eta^{\prime}\left(c_{i}^{\prime}\right)=\alpha$ for odd index $i$ and $\eta^{\prime}\left(c_{j}^{\prime}\right)=1-\alpha$ for even index $j$, where $1 \leq i, j \leq t+1$. These two choices of $\alpha$, together with the possible values 0 or 1 for $\eta^{\prime}\left(c_{0}^{\prime}\right)$ and $\eta^{\prime}\left(c_{t+2}^{\prime}\right)$, yield the following eight possibilities for the epimorphism $\eta^{\prime}=\eta_{\alpha, \beta, \delta}^{\prime}$ where $\alpha, \beta, \delta \in\{0,1\}$ :

$$
\eta_{\alpha, \beta, \delta}^{\prime}\left(c_{i}^{\prime}\right)=\left\{\begin{array}{cl}
\beta & \text { for } i=0 \\
\alpha & \text { for } 1 \leq i \leq t+1, i \text { odd } \\
1-\alpha & \text { for } 1 \leq i \leq t+1, i \text { even } \\
\delta & \text { for } i=t+2
\end{array}\right.
$$

Riemann-Hurwitz formula and [7, Thms. 2.2.4, 2.3.3, Cor. 2.3.4] yield finally that the signature of $\Gamma^{\prime}=\operatorname{ker} \eta_{\alpha, \beta, \delta}^{\prime}$ is:

- if $t$ is even, then

$$
\begin{aligned}
& \sigma\left(\operatorname{ker} \eta_{0,0,0}^{\prime}\right)=\left(0 ;+;[-] ;\left\{(-),{ }^{(t / 2)}{ }^{-1},(-),(2, n / 2,2,2, n / 2,2)\right\}\right), \\
& \sigma\left(\operatorname{ker} \eta_{0,0,1}^{\prime}\right)=\sigma\left(\operatorname{ker} \eta_{0,1,0}^{\prime}\right)=\left(0 ;+;[-] ;\left\{(-),{ }^{t / 2} .{ }^{\prime},(-),(2,2, n / 4)\right\}\right) \text {, } \\
& \sigma\left(\operatorname{ker} \eta_{0,1,1}^{\prime}\right)=\left(0 ;+;[n / 2] ;\left\{(-),{ }^{(t / 2)+1}+(-)\right\}\right) \text {, } \\
& \sigma\left(\operatorname{ker} \eta_{1,0,0}^{\prime}\right)=\left(0 ;+;[-] ;\left\{(-),{ }_{.}^{t / 2},(-),(n / 2, n / 2)\right\}\right), \\
& \sigma\left(\operatorname{ker} \eta_{1,0,1}^{\prime}\right)=\sigma\left(\operatorname{ker} \eta_{1,1,0}^{\prime}\right)=\left(0 ;+;[2] ;\left\{(-),{ }^{t / 2} .,(-),(n / 4)\right\}\right) \text {, } \\
& \sigma\left(\operatorname{ker} \eta_{1,1,1}^{\prime}\right)=(0 ;+;[2,2, n / 2] ;\{(-), \stackrel{t / 2}{.},(-)\}) \text {; }
\end{aligned}
$$


- if $t$ is odd, then

$$
\begin{aligned}
& \sigma\left(\operatorname{ker} \eta_{0,0,0}^{\prime}\right)=\sigma\left(\operatorname{ker} \eta_{1,0,0}^{\prime}\right)=\left(0 ;+;[-] ;\left\{(-),{ }^{(t-1) / 2},(-),(2,2, n / 2, n / 2)\right\}\right), \\
& \sigma\left(\operatorname{ker} \eta_{0,0,1}^{\prime}\right)=\sigma\left(\operatorname{ker} \eta_{1,1,0}^{\prime}\right)=\left(0 ;+;[2] ;\left\{(-),{ }^{(t-1) / 2},(-),(2,2, n / 4)\right\}\right), \\
& \sigma\left(\operatorname{ker} \eta_{0,1,0}^{\prime}\right)=\sigma\left(\operatorname{ker} \eta_{1,0,1}^{\prime}\right)=\left(0 ;+;[-] ;\left\{(-),{ }^{(t+1) / 2},(-),(n / 4)\right\}\right), \\
& \sigma\left(\operatorname{ker} \eta_{0,1,1}^{\prime}\right)=\sigma\left(\operatorname{ker} \eta_{1,1,1}^{\prime}\right)=\left(0 ;+;[2, n / 2] ;\left\{(-),{ }^{(t+1) / 2},(-)\right\}\right) .
\end{aligned}
$$

All these signatures having topological genus zero, the surface $X^{\prime}=U / \Gamma^{\prime}$ is planar. As to the number of its boundary components, it is either $t / 2$ or $t / 2+1$ if $t$ is even, that is, if $n$ divides $g+1$, and either $(t+1) / 2$ or $(t+3) / 2$ otherwise.

Cyclic and dihedral $n$-sheeted coverings with $n>2$ have been treated separately in Theorems 3.3 and 3.5. respectively. However, for $n=2$ the groups $C_{n}$ and $D_{n / 2}$ coincide and this is the reason why the result for double coverings requires a separate statement. This is the content of Theorem 3.6. whose proof is omitted since it can be easily extracted from both theorems; see also [5], where double coverings are studied to its full extent.

3.6. Theorem. Let $X$ be a hyperelliptic, planar, compact, Klein surface of algebraic genus $g \geq 2$ and let $u$ be an involution on $X$ different from its hyperelliptic involution. Let $\pi: X \rightarrow X^{\prime}$ with $X^{\prime}=X /\langle u\rangle$ be the induced double covering. Then the Klein surface $X^{\prime}$ is hyperelliptic and either planar or a projective plane with holes. Moreover, the number of its boundary components is

(a) $(g+1) / 2$ if $X^{\prime}$ is a projective plane with holes, or

(b) $(g+\varepsilon) / 2$ with $\varepsilon=1,2,3$ or 4 otherwise.

All the possibilities occur for different actions of $u$ whenever the value of the number of boundary components is an integer.

\section{Algebraic models}

Our goal in this section is to describe in algebraic terms the coverings $\pi: X \rightarrow X^{\prime}$ studied in a combinatorial way in the previous one. To that end we prefer to slightly change the terminology. In what follows we shall use the same term complex algebraic curve to refer to an affine algebraic curve defined over the field $\mathbb{C}$ of complex numbers, its projective non-singular model and its associated compact Riemann surface. Also, the expressions rational map and Riemann surface morphism will be used as synonymous.

In order to deal with algebraic models, it is profitable to understand Klein surfaces as symmetric Riemann surfaces. A symmetric Riemann surface, or real algebraic curve, is a pair $(S, \tau)$ where $S$ is a complex algebraic curve and $\tau$ is a symmetry of $S$, that is, an antianalytic involution $\tau: S \rightarrow S$. Each connected component of the set $\operatorname{Fix}(\tau)$ of fixed points of $\tau$ is called an oval, in Hilbert's terminology.

A rational map between two real algebraic curves $(S, \tau)$ and $\left(S^{\prime}, \tau^{\prime}\right)$ is a rational map $f: S \rightarrow S^{\prime}$ of complex curves such that $f \circ \tau=\tau^{\prime} \circ f$. In particular, a covering $(S, \tau) \rightarrow\left(S^{\prime}, \tau^{\prime}\right)$ is a covering of complex curves $\pi: S \rightarrow S^{\prime}$ such that $\pi \circ \tau=\tau^{\prime} \circ \pi$.

Given a real algebraic curve $(S, \tau)$, the quotient $S /\langle\tau\rangle$ is a compact Klein surface and the assignment $(S, \tau) \rightarrow S /\langle\tau\rangle$ induces a functorial equivalence between real algebraic curves and compact Klein surfaces; see [3]. Under this equivalence, the set of all birational automorphisms of $(S, \tau)$ constitutes a group canonically isomorphic to the automorphism group of the Klein surface $S /\langle\tau\rangle$. Of course, we will also use 
as synonymous the terms, real algebraic curve, its projective non-singular model, and its associated compact Klein surface.

This way, giving an algebraic model of a covering $\pi:(S, \tau) \rightarrow\left(S^{\prime}, \tau^{\prime}\right)$ amounts to providing defining polynomial equations for both $S$ and $S^{\prime}$, and rational formulae for generators of the covering transformation group $G$, and for the symmetries $\tau$ and $\tau^{\prime}$, and for the covering projection $\pi$. These are provided by Theorems 4.3 and 4.5 ,

We stress that it is the presence of symmetries that makes the search of algebraic models more involved than in the classical context.

Before dealing with symmetries on complex curves, we first set some preparatory material concerning just hyperelliptic complex curves.

It is well known that a hyperelliptic complex curve of genus $g \geq 2$ can be represented by the affine plane model

$$
\left\{(x, y) \in \mathbb{C}^{2}: y^{2}=P(x)\right\}
$$

where $P(x)$ is a monic polynomial of degree $2 g+1$ or $2 g+2$ without multiple roots. Its projective closure is obtained by adding either one or two extra points according to the parity of the degree of $P$. We call them the points at infinity of the chosen model, the finite ones being the points in $\mathbb{C}^{2}$. The hyperelliptic involution, which acts as $(x, y) \mapsto(x,-y)$ on the finite points, fixes the unique point at infinity if $P$ has odd degree and interchanges the points at infinity otherwise. The fixed points of the hyperelliptic involution are the Weierstrass points of the curve, and the first coordinates of the finite ones are the roots of $P(x)$; therefore, they determine a polynomial equation of the curve.

Consequently, given any covering $\pi: S \rightarrow S^{\prime}$ between hyperelliptic complex curves, in order to get algebraic models of $S$ and $S^{\prime}$ it is necessary to study the relation between the sets $W(S)$ and $W\left(S^{\prime}\right)$ of Weierstrass points of $S$ and $S^{\prime}$ respectively. This last set contains, often properly, the image of $W(S)$ under the covering $\pi: S \rightarrow S^{\prime}$; see Remark 3.4. In fact, the following holds.

4.1. Lemma. Let $\pi: S \rightarrow S^{\prime}$ be a normal branched covering between complex algebraic curves of genera $\geq 2$ and let $G$ be the covering transformation group. Assume that $S$ is hyperelliptic and that $G$ does not contain the hyperelliptic involution $h$ of S. Then

(i) the curve $S^{\prime}$ is also hyperelliptic;

(ii) the set $W\left(S^{\prime}\right)$ of Weierstrass points of $S^{\prime}$ is the union

$$
W\left(S^{\prime}\right)=\pi(W(S)) \cup \pi\left(\bigcup_{\gamma \in G-\{i d\}} \operatorname{Fix}(\gamma \circ h)\right) .
$$

Proof. It is easy to check that the equality $h^{\prime} \circ \pi=\pi \circ h$ defines an automorphism $h^{\prime}$ of $S^{\prime}$, which is in fact an involution since $h \notin G$. Moreover, this equality also allows us to define an obvious morphism from $S /\langle h\rangle$ onto $S^{\prime} /\left\langle h^{\prime}\right\rangle$. Since $S /\langle h\rangle$ has genus zero, the same holds true for $S^{\prime} /\left\langle h^{\prime}\right\rangle$. This shows that $S^{\prime}$ is hyperelliptic and that $h^{\prime}$ is its hyperelliptic involution. 
To show the equality in (ii), we calculate the fixed points of $h^{\prime}$. Let $p^{\prime}=\pi(p)$ be a point in $S^{\prime}$. Then

$$
\begin{aligned}
h^{\prime}\left(p^{\prime}\right)=p^{\prime} & \Longleftrightarrow h^{\prime}(\pi(p))=\pi(p) \Longleftrightarrow \pi(h(p))=\pi(p) \\
& \Longleftrightarrow \quad \text { there exists } \gamma \in G \text { such that } p=(\gamma \circ h)(p) .
\end{aligned}
$$

Therefore $W\left(S^{\prime}\right)=\pi\left(\bigcup_{\gamma \in G} \operatorname{Fix}(\gamma \circ h)\right)$. This is the equality in part (ii).

Birational isomorphisms between hyperelliptic complex algebraic curves are closely related to automorphisms of the Riemann sphere. We now recall how this relation works in terms of algebraic equations. Let

$$
\left\{(x, y) \in \mathbb{C}^{2}: y^{2}=P_{1}(x)\right\} \quad \text { and } \quad\left\{(x, y) \in \mathbb{C}^{2}: y^{2}=P_{2}(x)\right\}
$$

be affine plane models of two hyperelliptic complex curves $R_{1}$ and $R_{2}$. The branch point set $B_{i}$ of $R_{i}$ is the set of complex roots of the polynomial $P_{i}$ plus $\infty$ if $P_{i}$ has odd degree. Every birational isomorphism $\varphi: R_{1} \rightarrow R_{2}$ induces a Möbius transformation $\widehat{\varphi}: \widehat{\mathbb{C}} \rightarrow \widehat{\mathbb{C}}$ which maps $B_{1}$ onto $B_{2}$. In fact, $\widehat{\varphi}$ is defined by $\widehat{\varphi}:$ $\pi_{1}(p) \mapsto \pi_{2}(\varphi(p))$ where $\pi_{i}: R_{i} \rightarrow \widehat{\mathbb{C}}:(x, y) \mapsto x$. Moreover, the symmetry $s$ of a real algebraic curve $(R, s)$ induces an anti-analytic Möbius transformation $\widehat{s}$ which preserves the branch point set of $R$.

Conversely, every Möbius transformation $m: \widehat{\mathbb{C}} \rightarrow \widehat{\mathbb{C}}$ which maps $B_{1}$ onto $B_{2}$ induces exactly two isomorphisms $\varphi_{1}, \varphi_{2}: R_{1} \rightarrow R_{2}$ such that $\widehat{\varphi}_{i}=m$. Indeed, $\varphi_{2}=\varphi_{1} \circ h$, where $h$ is the hyperelliptic involution of $R_{1}$. The precise formulae of the liftings $\varphi_{1}, \varphi_{2}$ of $m$ are given in [8].

Non-necessarily bijective rational maps between two hyperelliptic complex curves also induce morphisms of the Riemann sphere onto itself defined in the obvious way. In particular, for the $n$-sheeted covering $\pi: S \rightarrow S^{\prime}$, we will denote by $\widehat{\pi}: \widehat{\mathbb{C}} \rightarrow \widehat{\mathbb{C}}$ the $n: 1$ meromorphic function induced by $\pi$.

The topology of a hyperelliptic real algebraic curve $(R, s)$ is determined by the number of branch points of $R$ fixed by the induced involution $\widehat{s}$. For the purposes of this paper the following lemma is useful (for a proof in a more general setting, see, e.g. [13, Prop. 6.3]). In the terminology of real algebraic geometry, planar real algebraic curves are called M-curves, while projective planes with holes are called $(\mathrm{M}-1)$-curves. We will use this terminology in the next lemma and in the rest of this section.

4.2. Lemma. With the above notations, let $2 k$ be the number of branch points of $R$ fixed by the induced involution $\widehat{s}$ and let $g$ be the genus of $R$. If $k>0$, then the real algebraic curve $(R, s)$ has $k$ ovals. In particular,

(i) $(R, s)$ is an $M$-curve if and only if $k=g+1$;

(ii) $(R, s)$ is an $(M-1)$-curve if and only if $k=g$.

We now describe algebraically the coverings studied from a combinatorial point of view in the previous section. We begin with cyclic coverings of degree $n>2$. We already proved in Theorem 3.3, that in this case $n$ divides $g+1$.

4.3. Theorem. Let $\pi:(S, \tau) \rightarrow\left(S^{\prime}, \tau^{\prime}\right)$ be a normal, branched, cyclic, $n$-sheeted covering where $(S, \tau)$ is a hyperelliptic real algebraic $M$-curve of algebraic genus $g \geq 2$. Assume that $n>2$ and that the covering transformation group $G$ does not contain the hyperelliptic involution of $S$. Then, up to dianalytic equivalence, the 
covering $\pi:(S, \tau) \rightarrow\left(S^{\prime}, \tau^{\prime}\right)$, can be represented in terms of algebraic equations as follows:

$$
S: w^{2}=\prod_{j=1}^{(2 g+2) / n}\left(z^{n}-a_{j}^{n}\right) \quad \text { and } \quad \tau(z, w)=\left(\frac{1}{\bar{z}}, \frac{\bar{w}}{\bar{z}^{g+1}}\right),
$$

for some unimodular complex numbers $a_{j}$ such that $\prod_{j=1}^{(2 g+2) / n} a_{j}^{n}=1$ and $a_{j}^{n} \neq a_{i}^{n}$ for $i \neq j$. The group $G$ is generated by the automorphism

$u_{\varepsilon}:(z, w) \rightarrow\left(z e^{2 \pi i / n},(-1)^{\varepsilon} w\right)$, where either $\varepsilon=0$ (whenever $n$ is odd) or $\varepsilon=1$.

For each value of $\varepsilon$ the covered curve $\left(S^{\prime}, \tau^{\prime}\right)$ is given by

$$
S^{\prime}: W^{2}=Z^{\varepsilon} \prod_{j=1}^{(2 g+2) / n}\left(Z-a_{j}^{n}\right), \quad \tau^{\prime}(Z, W)=\left(\frac{1}{\bar{Z}}, \frac{\bar{W}}{\bar{Z}^{\varepsilon+(g+1) / n}}\right)
$$

and the covering projection is given by

$$
\pi:(z, w) \mapsto(Z, W)=\left(z^{n}, z^{\varepsilon n / 2} w\right) .
$$

Proof. We begin by showing that $S$ and $\tau$ can be represented in terms of algebraic equations as in (1). Let $u$ be a generator of the cyclic group $G$. The automorphism $\widehat{u}$ of the Riemann sphere $\widehat{\mathbb{C}}$ induced by $u$ also has order $n$ since $\langle u\rangle$ does not contain the hyperelliptic involution $h$ of $S$. We may assume, after conjugation by a Möbius transformation, that $\widehat{u}$ fixes 0 and $\infty$ and so $\widehat{u}(z)=\xi_{n} z$, where $\xi_{n}=e^{2 \pi i / n}$. As to the antianalytic involution $\widehat{\tau}$ induced by $\tau$, it fixes points and commutes with $\widehat{u}$. Again, after conjugation by a Möbius transformation which commutes with $\widehat{u}$, we may assume that $\widehat{\tau}(z)=1 / \bar{z}$. Therefore, since the branch point set of $S$ is invariant under the action of $\widehat{u}$ and pointwise fixed by $\widehat{\tau}$ (see Lemma 4.2), there exist unimodular complex numbers $a_{j}$ such that $S$ admits an equation of the form

$$
S: w^{2}=\prod_{j=1}^{(2 g+2) / n}\left(z^{n}-a_{j}^{n}\right) \quad \text { with } a_{j}^{n} \neq a_{i}^{n} \text { for } i \neq j .
$$

It only remains to get the condition $\prod_{j=1}^{(2 g+2) / n} a_{j}^{n}=1$. This can be achieved by rotating the branch points an appropriate angle $\theta$. Indeed, we can choose $\theta=$ $-\theta_{S} /(g+1)$, where $\theta_{S} \in[0, \pi)$ is defined by the condition $\prod_{j=1}^{(2 g+2) / n} a_{j}^{n}=e^{2 i \theta_{S}}$. This way, the rotated branch points $b_{j}=a_{j} e^{i \theta}$ are also unimodular and satisfy the condition $\prod_{j=1}^{(2 g+2) / n} b_{j}^{n}=1$. They are also permuted by $\widehat{u}$ since the rotation $z \mapsto z e^{i \theta}$ commutes with $\widehat{u}$. Therefore, we may assume from the beginning that $S$ can be represented as in (11).

The rotation $z \mapsto z e^{i \theta}$ also commutes with $\widehat{\tau}: z \mapsto 1 / \bar{z}$, and so the symmetry $\tau$ in $S$ is one of the two liftings of $\widehat{\tau}$. From the equation of $S$ it follows easily that such liftings are $\tau_{1}$ and $\tau_{1} \circ h$, where

$$
\tau_{1}:(z, w) \mapsto\left(1 / \bar{z}, \bar{w} / \bar{z}^{g+1}\right) .
$$

If $\tau=\tau_{1}$, then the claim concerning the algebraic equations of $S$ and $\tau$ is proved. If not, we choose $\alpha_{j}=a_{j} e^{i \pi /(g+1)}$ and substitute the real algebraic curve $\left(S, \tau_{1} \circ h\right)$ by $\left(T, \tau_{1}\right)$ where

$$
T: w^{2}=\prod_{j=1}^{(2 g+2) / n}\left(z^{n}-\alpha_{j}^{n}\right)
$$


Indeed, these two real algebraic curves $\left(S, \tau_{1} \circ h\right)$ and $\left(T, \tau_{1}\right)$ are isomorphic via $(z, w) \mapsto\left(z e^{i \pi /(g+1)}, w\right)$. Observe that $\left|\alpha_{j}\right|=1$ for all $j, \alpha_{i}^{n} \neq \alpha_{j}^{n}$ if $i \neq j$ and $\prod_{j=1}^{(2 g+2) / n} \alpha_{j}^{n}=1$.

Once we have the real algebraic curve $(S, \tau)$ represented as in (1), the formula of a generator $u$ of $G$ can be explicitly calculated. Indeed, it is a lifting of $\widehat{u}$ and so there exists $\varepsilon \in\{0,1\}$ such that

$$
u=u_{\varepsilon}:(z, w) \rightarrow\left(z e^{2 \pi i / n},(-1)^{\varepsilon} w\right)
$$

Clearly, if the order $n$ of $u_{\varepsilon}$ is odd, then $\varepsilon$ has to be 0 . We stress the fact that $u_{\varepsilon}$ is an automorphism of the real algebraic curve $(S, \tau)$ for both values of $\varepsilon$ since it commutes with $\tau$.

In order to obtain a polynomial equation $W^{2}=P(Z)$ of $S^{\prime}$ we calculate the set $W\left(S^{\prime}\right)$ of its Weierstrass points, and for that we use Lemma 4.1. We first look for a formula of the $n: 1$ meromorphic function $\widehat{\pi}: \widehat{\mathbb{C}} \rightarrow \widehat{\mathbb{C}}$ induced by $\pi$. It follows easily that $\widehat{\pi}$ is constant on each $\langle\widehat{u}\rangle$-orbit. Since the fixed points of $\widehat{u}$ are 0 and $\infty$, these are ramifications points of $\widehat{\pi}$, both of multiplicity $n$. Thus, after composing with a Möbius transformation if necessary, we may assume that $z=0$ is the unique zero and $z=\infty$ is the unique pole of $\widehat{\pi}$, both of order $n$. Hence, $\widehat{\pi}(z)=\lambda z^{n}$ for some $\lambda \in \mathbb{C}-\{0\}$ and we may assume $\lambda=1$ after composing again with a Möbius transformation if necessary. This suffices to compute $\pi(W(S))$. Indeed, since

$$
W(S)=\left\{\left(\xi_{n}^{k} a_{j}, 0\right): k=0, \ldots, n-1 ; j=1, \ldots,(2 g+2) / n\right\}
$$

we get

$$
\pi(W(S))=\left\{\left(a_{j}^{n}, 0\right): j=1, \ldots,(2 g+2) / n\right\}
$$

where we have used that the second coordinate of a finite Weierstrass point is zero because it is fixed by the hyperelliptic involution $(x, y) \mapsto(x,-y)$. This yields $(2 g+2) / n$ out of the $2 g^{\prime}+2$ Weierstrass points of the algebraic curve $S^{\prime}$, where $g^{\prime}$ is the genus of $S^{\prime}$. We now distinguish two cases according to $\varepsilon=0$ or 1 in the formula of the generator $u$ of $G$.

In the first case it is $u(z, w)=\left(\xi_{n} z, w\right)$, all of whose non-trivial powers have four fixed points in $S$, each with multiplicity $n$. Then $2 g^{\prime}+2=(2 g+2) / n$ by Riemann-Hurwitz formula. Hence, the above $(2 g+2) / n$ points in $\pi(W(S))$ are all of the Weierstrass points of $S^{\prime}$. Consequently, we get the following defining equation for $S^{\prime}$ :

$$
S^{\prime}: W^{2}=\prod_{j=1}^{(2 g+2) / n}\left(Z-a_{j}^{n}\right) .
$$

So a formula for $\pi$ has the form $\pi(z, w)=\left(z^{n}, f(z, w)\right)$ for some rational function $f$ such that

$$
f^{2}=\prod_{j=1}^{(2 g+2) / n}\left(z^{n}-a_{j}^{n}\right)=w^{2} .
$$

Hence, up to automorphism of $S^{\prime}$,

$$
\pi:(z, w) \mapsto(Z, W)=\left(z^{n}, w\right)
$$


As to the anti-analytic involution $\tau^{\prime}$, notice that it is determined by the equality $\pi \circ \tau=\tau^{\prime} \circ \pi$. In fact, $\widehat{\pi} \circ \widehat{\tau}=\widehat{\tau}^{\prime} \circ \widehat{\pi}$ yields $\widehat{\tau}^{\prime}(Z)=1 / \bar{Z}$, and so

$$
\tau^{\prime}(Z, W)=\left(1 / \bar{Z}, \delta \bar{W} / \bar{Z}^{(g+1) / n}\right) \quad \text { where } \delta=1 \text { or } \delta=-1 .
$$

The right value of $\delta$ is deduced from $\pi \circ \tau=\tau^{\prime} \circ \pi$. In this case we get $\delta=1$.

We now deal with the second case $\varepsilon=1$, that is, $u:(z, w) \mapsto\left(\xi_{n} z,-w\right)$. Recall that in this case $n$ has to be even.

Let us calculate $W\left(S^{\prime}\right)$. For each $k=1, \ldots, n-1$, the set in $S$ of finite fixed points of $u^{k} \circ h:(z, w) \mapsto\left(\xi_{n}^{k} z,(-1)^{k+1} w\right)$ is either empty if $k$ is even or $\{(0,1),(0,-1)\}$ if $k$ is odd. Moreover, $\pi$ maps these two points to the same Weierstrass point $(0,0)$ of $S^{\prime}$ because $u(0,1)=(0,-1)$ and $\widehat{\pi}(0)=0$. Thus, the set of finite Weierstrass points of $S^{\prime}$ is the union

$$
W\left(S^{\prime}\right)=\pi(W(S)) \cup\{(0,0)\}=\left\{\left(a_{j}^{n}, 0\right): j=1, \ldots,(2 g+2) / n\right\} \cup\{(0,0)\},
$$

and so we get the following defining equation for $S^{\prime}$ :

$$
S^{\prime}: W^{2}=Z \prod_{j=1}^{(2 g+2) / n}\left(Z-a_{j}^{n}\right) .
$$

Finally, the covering projection $\pi$ and the antianalytic involution $\tau^{\prime}$ are given by

$$
\pi:(z, w) \mapsto(Z, W)=\left(z^{n}, z^{n / 2} w\right) \text { and } \tau^{\prime}(Z, W)=\left(1 / \bar{Z}, \bar{W} / \bar{Z}^{1+(g+1) / n}\right),
$$

as one can easily obtain arguing as in the first case.

4.4. Remark. With the algebraic formulae at hand one can describe explicitly all ramification data and topological features of the cyclic coverings studied in the previous theorem. For example, the topological type of the covered curve $\left(S^{\prime}, \tau^{\prime}\right)$ is determined, according to Lemma 4.2. by the number of branch points of $S^{\prime}$ fixed by $\widehat{\tau}^{\prime}$. In our context, the set of fixed points of $\widehat{\tau}^{\prime}$ is the unit circle. In case $\varepsilon=0$ in the above theorem, all of the branch points of $S^{\prime}$ lie in the unit circle and so $\left(S^{\prime}, \tau^{\prime}\right)$ is an M-curve; however, if $\varepsilon=1$, then $\left(S^{\prime}, \tau^{\prime}\right)$ is an (M-1)-curve since all but two of the branch points of $S^{\prime}$, namely 0 and $\infty$, lie in the unit circle.

As a consequence, with the notations in Theorem 3.3, the NEC group $\Gamma^{\prime}$ such that the Klein surface $S^{\prime} /\left\langle\tau^{\prime}\right\rangle$ is isomorphic to $U / \Gamma^{\prime}$ has signature either $\sigma_{1}=$ $\left(0 ;+;[n, n] ;\left\{(-),{ }^{(g+1)} /{ }^{\prime},(-)\right\}\right)$ or $\sigma_{2}=(1 ;-;[n / 2] ;\{(-),(g+1) / n,(-)\})$, according to $G$ be generated by $u_{0}$ or $u_{1}$ respectively. As observed in Remark 3.4 the projection $\pi: S /\langle\tau\rangle \rightarrow S^{\prime} /\left\langle\tau^{\prime}\right\rangle$ ramifies just over two interior points (with branching order $n$ ) in the first case, and just over one interior point (with branching order $n / 2$ ), in the second case. Let us find the coordinates of these points in our models. All non-trivial powers of $u_{0}$ fix the same four points in $S$, namely, $(0,1),(0,-1)$ and the two points at infinity, and $\pi$ has multiplicity $n$ at all of them. In the Klein surface $S /\langle\tau\rangle$ these four points are pairwise identified, which yields the two ramification points of the projection $\pi: S /\langle\tau\rangle \rightarrow S^{\prime} /\left\langle\tau^{\prime}\right\rangle$. These two points are indeed projected over two interior points of $S^{\prime} /\left\langle\tau^{\prime}\right\rangle$, since $\tau^{\prime}$ fixes neither $\pi(0,1)=(0,1)$ nor $\pi(0,-1)=(0,-1)$. Here we are using that the boundary points of $S^{\prime} /\left\langle\tau^{\prime}\right\rangle$ are the $\left\langle\tau^{\prime}\right\rangle$-orbits of points in $S^{\prime}$ fixed by $\tau^{\prime}$.

On the contrary, the odd powers of $u_{1}$ fix no point of $S$, whilst its non-trivial even powers fix the same four points in $S$, namely, $(0,1),(0,-1)$ and the two points at infinity, with $\pi$ having now multiplicity $n / 2$ at all of them. In the Klein surface 
$S /\langle\tau\rangle$ these four points are pairwise identified, which yields two ramification points of the projection $\pi: S /\langle\tau\rangle \rightarrow S^{\prime} /\left\langle\tau^{\prime}\right\rangle$. These two points are projected over the same point of $S^{\prime} /\left\langle\tau^{\prime}\right\rangle$, namely, $\pi(0,1)=\pi(0,-1)=(0,0)$. Moreover, this projected point is indeed interior in $S^{\prime} /\left\langle\tau^{\prime}\right\rangle$ since it is not fixed by $\tau^{\prime}$.

We now deal with dihedral $n$-sheeted coverings with $n>2$. Recall that in this case $n$ is an even divisor of $2 g+2$; see Proposition 3.2.

4.5. Theorem. Let $\pi:(S, \tau) \rightarrow\left(S^{\prime}, \tau^{\prime}\right)$ be a normal, branched, dihedral, n-sheeted covering where $(S, \tau)$ is a hyperelliptic real algebraic $M$-curve of algebraic genus $g \geq 2$. Assume that $n=2 m>2$ and that the covering transformation group $G$ does not contain the hyperelliptic involution of $S$. Then there exist $\varepsilon_{1}, \varepsilon_{2}, \varepsilon_{3} \in\{-1,+1\}$, with $\varepsilon_{2}=1$ whenever $m$ is odd, such that, up to dianalytic equivalence, the covering $\pi:(S, \tau) \rightarrow\left(S^{\prime}, \tau^{\prime}\right)$, can be represented in terms of algebraic equations as follows:

$$
S: w^{2}=\prod_{j=1}^{(g+1) / m}\left(z^{m}-a_{j}^{m}\right)\left(z^{m}-\bar{a}_{j}^{m}\right) \quad \text { and } \quad \tau(z, w)=\left(\frac{1}{\bar{z}}, \frac{\varepsilon_{1} \bar{w}}{\bar{z}^{g+1}}\right),
$$

for some unimodular complex numbers $a_{j}$ such that the polynomial in the variable $z$ on the right-hand side has no multiple roots. The covering transformation group is generated by the automorphisms $u$ and $v$, where

$$
u:(z, w) \rightarrow\left(z e^{2 \pi i / m}, \varepsilon_{2} w\right) \quad \text { and } \quad v:(z, w) \rightarrow\left(\frac{1}{z}, \frac{\varepsilon_{3} w}{z^{g+1}}\right) .
$$

Let $b_{j}=a_{j}^{m}+\bar{a}_{j}{ }^{m}$ for all $j$. Then, the covered curve $\left(S^{\prime}, \tau^{\prime}\right)$ and the projection $\pi$ are given by

$$
S^{\prime}: W^{2}=(Z-2)^{\varepsilon_{4}}(Z+2)^{\varepsilon_{5}} \prod_{j=1}^{(g+1) / m}\left(Z-b_{j}\right), \quad \tau^{\prime}(Z, W)=\left(\bar{Z}, \varepsilon_{1} \varepsilon_{3} \bar{W}\right),
$$

and

$$
\pi(z, w)=(Z, W)=\left(z^{m}+\frac{1}{z^{m}}, \frac{w\left(z^{m}-1\right)^{\varepsilon_{4}}\left(z^{m}+1\right)^{\varepsilon_{5}}}{z^{\left(m\left(\varepsilon_{4}+\varepsilon_{5}\right)+g+1\right) / 2}}\right),
$$

where $\varepsilon_{4}, \varepsilon_{5} \in\{0,1\}$ with

$$
\varepsilon_{4}=0 \Longleftrightarrow \varepsilon_{3}=1 \quad \text { and } \quad \varepsilon_{5}=0 \Longleftrightarrow \varepsilon_{2} \cdot \varepsilon_{3}=(-1)^{(g+1) / m} .
$$

Proof. We begin by showing that $S$ and $\tau$ can be represented in terms of algebraic equations as in (2). Let $u, v \in G$ be generators of the dihedral group $G$ of orders $m$ and 2 respectively. The automorphisms $\widehat{u}$ and $\widehat{v}$ of the Riemann sphere $\widehat{\mathbb{C}}$ induced by $u$ and $v$ also generate a dihedral group of order $2 m$ since $G$ does not contain the hyperelliptic involution $h$. We may assume, after conjugation by a Möbius transformation if necessary, that $\widehat{u}$ fixes 0 and $\infty$ and that $\widehat{v}$ fixes 1 . It follows that $\widehat{u}(z)=\xi_{m} z$, where $\xi_{m}=e^{2 \pi i / m}$, and $\widehat{v}(z)=1 / z$. The anti-analytic involution $\widehat{\tau}$ induced by $\tau$ fixes points and commutes with $\widehat{u}$ and $\widehat{v}$. Hence, if $m>2$, then $\widehat{\tau}(z)=1 / \bar{z}$. If $m=2$, the roles of $\widehat{u}$ and $\widehat{v}$ are interchangeable and we may assume again that $\widehat{\tau}(z)=1 / \bar{z}$. It is then easy to check that, under the action of $\langle\widehat{u}, \widehat{v}\rangle$, there are three types of orbits of complex numbers $a$ fixed by $\widehat{\tau}$, namely:

- the roots of $\left(z^{m}-a^{m}\right)\left(z^{m}-\bar{a}^{m}\right)$ if the $\langle\widehat{u}, \widehat{v}\rangle$-stabilizer of $a$ is trivial,

- the roots of $\left(z^{m}-1\right)$ if $\widehat{v}(a)=a$, and

- the roots of $\left(z^{m}+1\right)$ if $(\widehat{u} \circ \widehat{v})(a)=a$. 
Since $(S, \tau)$ is an M-curve, its branch points are fixed by $\widehat{\tau}$; see Lemma 4.2. Moreover, they constitute an invariant set under the action of $\langle\widehat{u}, \widehat{v}\rangle$ and therefore there exist $\alpha, \beta \in\{0,1\}$ and a positive integer $t$ such that $S$ admits an equation of the form

$$
S: w^{2}=\left(z^{m}-1\right)^{\alpha}\left(z^{m}+1\right)^{\beta} \prod_{j=1}^{t}\left(z^{m}-a_{j}^{m}\right)\left(z^{m}-\bar{a}_{j}^{m}\right)
$$

where $\left|a_{j}\right|=1$ for all $j$ and the polynomial on the right-hand side has no multiple roots. We have to show that $\alpha=\beta=0$, in which case $t=(g+1) / m$ (as stated in the theorem), because $2 g+2=m(\alpha+\beta+2 t)$. This equality shows that $\alpha+\beta$ is even, since $m$ divides $g+1$, and so, it suffices to check, e.g., that $\alpha=0$. We will prove that, otherwise, no lifting $v$ of $\widehat{v}$ commutes with $\tau$, and so $v$ is not an automorphism of the real algebraic curve $(S, \tau)$.

In fact, the above equation of $S$ and the formulae of $\widehat{\tau}, \widehat{u}$ and $\widehat{v}$ yield the formulae of the anti-analytic involution $\tau$ and the automorphisms $u$ and $v$, namely,

$\tau(z, w)=\left(\frac{1}{\bar{z}}, \frac{\varepsilon_{1} i^{\alpha} \bar{w}}{\bar{z}^{g+1}}\right), u:(z, w) \rightarrow\left(z e^{2 \pi i / m}, \varepsilon_{2} w\right)$ and $v:(z, w) \rightarrow\left(\frac{1}{z}, \frac{\varepsilon_{3} i^{\alpha} w}{z^{g+1}}\right)$,

with $\varepsilon_{1}, \varepsilon_{2}, \varepsilon_{3}$ equal to +1 or -1 , and $i=\sqrt{-1}$. It is easy to check that if $\alpha=1$, then $v$ does not commute with $\tau$. Therefore, $\alpha=\beta=0$ and the above formulae of $S, \tau, u$ and $v$ coincide with those in the statement. Moreover, $\varepsilon_{2}=1$ whenever $m$ is odd since $u$ has order $m$.

In order to obtain a polynomial equation of $S^{\prime}$ we calculate the set of its finite Weierstrass points, and for that we use Lemma 4.1. Let $\widehat{\pi}: \widehat{\mathbb{C}} \rightarrow \widehat{\mathbb{C}}$ be the $2 m: 1$ meromorphic function induced by the covering $\pi: S \rightarrow S^{\prime}$. Computing the points in $\widehat{\mathbb{C}}$ with non-trivial $\langle\widehat{u}, \widehat{v}\rangle$-stabilizer yields that there are exactly three fibers with less than $2 m$ elements, namely

$$
\widehat{\pi}^{-1}(a)=\{0, \infty\} ; \quad \widehat{\pi}^{-1}(b)=\left\{z \in \mathbb{C}: z^{m}=1\right\} ; \quad \widehat{\pi}^{-1}(c)=\left\{z \in \mathbb{C}: z^{m}=-1\right\},
$$

for some $a, b, c \in \widehat{\mathbb{C}}$. Of course, the branching orders of $\widehat{\pi}$ at $a, b$ and $c$ are $m, 2$ and 2 respectively. After composing with a Möbius transformation if necessary, we may assume that $a=\infty, b=2$ and $c=-2$, and this implies $\widehat{\pi}(z)=z^{m}+z^{-m}$. We can now compute the projection $\pi(W(S))$, where

$$
W(S)=\left\{\left(\xi_{m}^{k} a_{j}, 0\right),\left(\xi_{m}^{k} \bar{a}_{j}, 0\right): k=0, \ldots, m-1, j=1, \ldots, t\right\}
$$

is the set of Weierstrass points of $S$. For all $k=0, \ldots, m-1$, we get $\widehat{\pi}\left(\xi_{m}^{k} a_{j}\right)=$ $\widehat{\pi}\left(\xi_{m}^{k} \bar{a}_{j}\right)=a_{j}^{m}+\bar{a}_{j}^{m}$. So, denoting $b_{j}=a_{j}^{m}+\bar{a}_{j}^{m}$ we get

$$
\pi(W(S))=\left\{\left(b_{j}, 0\right): j=1, \ldots, t\right\} \quad \text { and } \quad S^{\prime}: W^{2}=Q(Z) \prod_{j=1}^{t}\left(Z-b_{j}\right),
$$

where $Q$ is a monic polynomial whose roots are those complex numbers $\widehat{\pi}(z)$, different from $b_{j}$ for all $j$, such that $(z, w) \in S$ is fixed by $\gamma \circ h$ for some $\gamma \in G-\{i d\}$. The non-trivial elements of $G$ are $\gamma_{k, j}=u^{k} \circ v^{j}$ with $(k, j) \neq(0,0)$. Composing with $h$ yields

$$
\left(\gamma_{k, j} \circ h\right)(z, w)=\left(\xi_{m}^{k} z^{1-2 j}, \frac{-\varepsilon_{2}^{k} \varepsilon_{3}^{j} w}{z^{j(g+1)}}\right) .
$$

If $(z, w)$ is fixed by $\gamma_{k, j} \circ h$, then $z^{2 j}=\xi_{m}^{k}$, because $z \neq 0$. Thus, necessarily, $j=1$ and $z^{2 m}=\left(\xi_{m}^{k}\right)^{m}=1$; that is, $z^{m}= \pm 1$ and $\widehat{\pi}(z)=z^{m}+z^{-m}= \pm 2$. 
This proves that $S^{\prime}$ admits an equation as in (3) and a case-by-case analysis shows that the occurrences of the roots -2 or 2 coincide with the ones provided by formula (4).

Let us now calculate the second coordinate $W$ of the projection $\pi$. Since the point $\left(z^{m}+z^{-m}, W\right) \in S^{\prime}$ whenever $(z, w) \in S$ we get

$$
\begin{aligned}
W^{2} & =\left(z^{m}+z^{-m}-2\right)^{\varepsilon_{4}}\left(z^{m}+z^{-m}+2\right)^{\varepsilon_{5}} \prod_{j=1}^{(g+1) / m}\left(z^{m}+z^{-m}-a_{j}^{m}-\bar{a}_{j}^{m}\right) \\
& =\frac{\left(z^{m}-1\right)^{2 \varepsilon_{4}}\left(z^{m}+1\right)^{2 \varepsilon_{5}}}{z^{m\left(\varepsilon_{4}+\varepsilon_{5}\right)}} \prod_{j=1}^{(g+1) / m} \frac{\left(z^{m}-a_{j}^{m}\right)\left(z^{m}-\bar{a}_{j}^{m}\right)}{z^{m}} \\
& =\left(\frac{\left(z^{m}-1\right)^{\varepsilon_{4}}\left(z^{m}+1\right)^{\varepsilon_{5}} w}{z^{\left(m\left(\varepsilon_{4}+\varepsilon_{5}\right)+g+1\right) / 2}}\right)^{2} .
\end{aligned}
$$

Using formula (4), it is easy to check that the exponent $m\left(\varepsilon_{4}+\varepsilon_{5}\right)+g+1$ is even and, therefore, up to automorphisms of $S^{\prime}$, the rational formula of the projection $\pi$ is as in the statement.

Finally, the formula of $\tau^{\prime}$ is obtained from the equalities $\pi \circ \tau=\tau^{\prime} \circ \pi$ and $\varepsilon_{3}=$ $(-1)^{\varepsilon_{4}}$.

4.6. Remarks. (i) The reader may have noticed that, unlike the cyclic case, there are two formulae for the symmetry $\tau$ in the statement of Theorem 4.5 according to $\varepsilon_{1}$ being +1 or -1 . Indeed, we are going to show that this is unavoidable if $(g+1) / m$ is even and the Weierstrass points of $S$ are in general position, in the sense that $(S, \tau)$ has no more automorphisms than those in $\langle G, h\rangle$.

Let us suppose, by the way of contradiction, that there exists an isomorphism $f:\left(S, \tau_{+1}\right) \rightarrow\left(S_{1}, \tau_{-1}\right)$ where the equations of $S$ and $S_{1}$ have the form required in Theorem 4.5 and $\tau_{\varepsilon_{1}}(z, w)=\left(1 / \bar{z}, \varepsilon_{1} \bar{w} / \bar{z}^{g+1}\right)$ with $\varepsilon_{1}=+1$ or -1 . We first deal with $m>2$.

The automorphism $f \circ u \circ f^{-1}$ in $S_{1}$ has order $m$ and so $f \circ u \circ f^{-1}=u^{\ell}$ for some integer $\ell$ coprime with $m$. Then $\widehat{f} \circ \widehat{u} \circ \widehat{f}^{-1}=\widehat{u}^{\ell}$ and this implies $\widehat{f}(\{0, \infty\})=$ $\{0, \infty\}$. So either $\widehat{f}(z)=\lambda z$ or $\widehat{f}(z)=\lambda / z$ for some $\lambda \neq 0$.

On the other hand, $\hat{f} \circ \hat{v} \circ \hat{f}^{-1}=\hat{u}^{k} \circ \widehat{v}$ for some $k \in\{1, \ldots, m\}$ and so $\hat{f}(1)=$ $\pm e^{k \pi i / m}$ since 1 is fixed by $\widehat{v}$ and $\pm e^{k \pi i / m}$ is fixed by $\widehat{u}^{k} \circ \widehat{v}$. Therefore $\widehat{f}$ has one of the following formulae:

$$
\widehat{f}(z)= \pm z e^{k \pi i / m} \quad \text { or } \quad \widehat{f}(z)= \pm e^{k \pi i / m} / z .
$$

The equations of $S$ and $S_{1}$ yield the formula of $f$ as a lifting of $\widehat{f}$.

- If $\widehat{f}(z)=z e^{k \pi i / m}$, then $f:(z, w) \mapsto\left(z e^{k \pi i / m}, \pm w\right)$. Thus

$$
f^{-1} \circ \tau_{-1} \circ f:(z, w)=\left(\frac{1}{\bar{z}}, \frac{-\bar{w} e^{k \pi i(g+1) / m}}{\bar{z}^{g+1}}\right)
$$

and so $f^{-1} \circ \tau_{-1} \circ f$ coincides with $\tau_{+1}$ if and only if $e^{k \pi i(g+1) / m}=-1$. This is false since $(g+1) / m$ is even.

- If $\widehat{f}(z)=-z e^{k \pi i / m}$, then $f:(z, w) \mapsto\left(-z e^{k \pi i / m}, \pm w\right)$. Thus

$$
f^{-1} \circ \tau_{-1} \circ f:(z, w)=\left(\frac{1}{\bar{z}}, \frac{-\bar{w}}{\bar{z}^{g+1}}\right)
$$

and so $f^{-1} \circ \tau_{-1} \circ f \neq \tau_{+1}$. 
- Finally, let us suppose that $\widehat{f}(z)= \pm e^{k \pi i / m} / z$. If $f^{-1} \circ \tau_{-1} \circ f=\tau_{+1}$, then

$$
(f \circ v)^{-1} \circ \tau_{-1} \circ(f \circ v)=\tau_{+1}
$$

since $v$ commutes with both symmetries $\tau_{-1}$ and $\tau_{+1}$. Then the map $g=$ $f \circ v$ would be an isomorphism between $\left(S, \tau_{+1}\right)$ and $\left(S_{1}, \tau_{-1}\right)$. However, the formula of $\widehat{g}$ is $\widehat{g}: z \mapsto \pm z e^{k \pi i / m}$, and we have just seen in the previous cases that this cannot happen.

If $m=2$, then $f \circ u \circ f^{-1}=u, v$ or $u v$ and we have to discard the last two possibilities since the first has just been considered. If $\widehat{f} \circ \widehat{u} \circ \widehat{f}^{-1}=\widehat{v}$ or $\widehat{u} \widehat{v}$, then $\widehat{f}$ would map $\{0, \infty\}$ onto the fixed points of $\widehat{v}$ or $\widehat{u} \widehat{v}$, which is impossible since these last lie on the unit circle and $\widehat{f}$ preserves it.

We point out that without the assumption about the general position of the Weierstrass points of $S$, the argument above fails, and in fact one can construct examples of curves $\left(S, \tau_{+1}\right)$ which are isomorphic to some $\left(S_{1}, \tau_{-1}\right)$.

In relation to this, if $(g+1) / m$ is odd and with any position of the Weierstrass points, then choosing $k=1$ in the first case discussed above yields an isomorphism $f:(z, w) \mapsto\left(z e^{\pi i / m}, w\right)$ between the real algebraic curves $\left(S, \tau_{+1}\right)$ and $\left(S_{1}, \tau_{-1}\right)$. Thus, under the condition $(g+1) / m$ odd, the real curves $\left(S_{1}, \tau_{-1}\right)$ can be avoided in the statement of Theorem 4.5 .

(ii) From the combinatorial study in Theorem 3.5 we know that $\left(S^{\prime}, \tau^{\prime}\right)$ is an M-curve. This follows also from the algebraic study in Theorem 4.5 because $\widehat{\tau}^{\prime}$ fixes all of the branch points of $S^{\prime}$ (see Lemma 4.2).

The whole ramification data and topological features of all dihedral coverings $\pi:(S, \tau) \rightarrow\left(S^{\prime}, \tau^{\prime}\right)$. To that end, it is useful to determine the signature $\sigma\left(\Gamma^{\prime}\right)$ of the NEC group $\Gamma^{\prime}$ such that $S^{\prime} /\left\langle\tau^{\prime}\right\rangle \simeq U / \Gamma^{\prime}$ occurring in the list of the proof of Theorem [3.5. The knowledge of such signature avoids a lot of calculations. The correspondence between such signature and the choice of values of $\varepsilon_{1}, \varepsilon_{2}, \varepsilon_{3}$ and the parity of $t=(g+1) / m$ in Theorem 4.5) is given in Table 1 in Proposition 4.7 below. We omit its tedious proof, but we develop in detail a case in Example 4.8.

4.7. Proposition. The correspondence mentioned above is given in Table 1 ,

4.8. Example. Suppose, with the notations in Theorem 4.5, that $\varepsilon_{1}=\varepsilon_{3}=1$ and $g$ is even. So both $m$ and $t$ are odd and $\varepsilon_{2}=1$. Hence, $\left(S^{\prime}, \tau^{\prime}\right)$ is defined by

$$
S^{\prime}: W^{2}=(Z+2) \prod_{j=1}^{t}\left(Z-b_{j}\right), \quad \tau^{\prime}(Z, W)=(\bar{Z}, \bar{W}) .
$$

Since both $m$ and $t$ are odd, it follows from the proof of Theorem 3.5 that the signature of the NEC group $\Gamma^{\prime}$ such that $S^{\prime} /\left\langle\tau^{\prime}\right\rangle \simeq U / \Gamma^{\prime}$ is either

$$
\sigma_{1}=\left(0 ;+;[-] ;\left\{(-),{ }^{(t-1)} \cdot{ }^{2} / 2,(-),(2,2, m, m)\right\}\right)
$$

or

$$
\sigma_{2}=\left(0 ;+;[2, m] ;\left\{(-),{ }^{(t+1) / 2},{ }^{2},(-)\right\}\right) .
$$

To decide which of them corresponds to our covering, note that although both signatures yield the existence of points with branching order 2 and $m$, they lie in the boundary of $S^{\prime} /\left\langle\tau^{\prime}\right\rangle$ in the first case, and in its interior in the second one. Those with branching order 2 are projections under $\pi$ of fixed points of the involution $v \in G$ of the algebraic curve $S$. Since $v$ is defined by $v:(z, w) \mapsto\left(1 / z, w / z^{g+1}\right)$, its fixed points in $S$ are $(1, \rho)$ and $(1,-\rho)$, where $\rho$ is the positive square root of 


\begin{tabular}{|c|c|c|c|c|}
\hline$\sigma\left(\Gamma^{\prime}\right)$ & $\varepsilon_{1}$ & $\varepsilon_{2}$ & $\varepsilon_{3}$ & $t=(g+1) / m$ \\
\hline$\left(0 ;+;[-] ;\left\{(-),{ }^{(t / 2)-1},(-),(2, m, 2,2, m, 2)\right\}\right)$ & +1 & +1 & +1 & even \\
\hline$\left(0 ;+;[-] ;\left\{(-),{ }^{(t-1)} \cdot{ }^{2},(-),(2,2, m, m)\right\}\right)$ & +1 & +1 & +1 & odd \\
\hline$\left(0 ;+;[m] ;\left\{(-),{ }^{(t / 2)+1},(-)\right\}\right)$ & +1 & +1 & -1 & even \\
\hline$\left.\left(0 ;+;[2, m] ;\left\{(-),{ }^{(t+1)} \cdot\right)^{2},(-)\right\}\right)$ & +1 & +1 & -1 & odd \\
\hline$(0 ;+;[-] ;\{(-), \stackrel{t / 2}{.},(-),(2,2, m / 2)\})$ & +1 & -1 & +1 & even \\
\hline$\left(0 ;+;[2] ;\left\{(-),{ }^{(t-1)} \cdot{ }^{2},(-),(2,2, m / 2)\right\}\right)$ & +1 & -1 & +1 & odd \\
\hline$(0 ;+;[-] ;\{(-), \stackrel{t / 2}{.},(-),(2,2, m / 2)\})$ & +1 & -1 & -1 & even \\
\hline$\left(0 ;+;[-] ;\left\{(-),{ }^{(t+1)} \cdot{ }^{2},(-),(m / 2)\right\}\right)$ & +1 & -1 & -1 & odd \\
\hline$\left(0 ;+;[2,2, m] ;\left\{(-),{ }^{t / 2} .,(-)\right\}\right)$ & -1 & +1 & +1 & even \\
\hline$\left(0 ;+;[2, m] ;\left\{(-),{ }^{(t+1)} \cdot{ }^{2},(-)\right\}\right)$ & -1 & +1 & +1 & odd \\
\hline$(0 ;+;[-] ;\{(-), \stackrel{t / 2}{.},(-),(m, m)\})$ & -1 & +1 & -1 & even \\
\hline$\left.\left(0 ;+;[-] ;\left\{(-),{ }^{(t-1)} \cdot\right)^{2},(-),(2,2, m, m)\right\}\right)$ & -1 & +1 & -1 & $o d d$ \\
\hline$(0 ;+;[2] ;\{(-), \stackrel{t / 2}{.},(-),(m / 2)\})$ & -1 & -1 & +1 & even \\
\hline$\left(0 ;+;[2] ;\left\{(-),{ }^{(t-1)} \cdot{ }^{2},(-),(2,2, m / 2)\right\}\right)$ & -1 & -1 & +1 & $o d d$ \\
\hline$(0 ;+;[2] ;\{(-), \stackrel{t / 2}{.},(-),(m / 2)\})$ & -1 & -1 & -1 & even \\
\hline$\left(0 ;+;[-] ;\left\{(-),{ }^{(t+1)} \cdot{ }^{\prime},(-),(m / 2)\right\}\right)$ & -1 & -1 & -1 & odd \\
\hline
\end{tabular}

TABLE 1. Algebraic-combinatorial correspondence

$\prod_{j=1}^{t}\left|1-a_{j}^{m}\right|^{2}$. So $p=\pi(1, \rho)$ is a branch point in $S^{\prime}$ of branching order 2. Since $\pi$ is given by

$$
\pi(z, w)=\left(z^{m}+\frac{1}{z^{m}}, \frac{w\left(z^{m}+1\right)}{z^{(m+g+1) / 2}}\right),
$$

one gets $p=(2,2 \rho)$, which is fixed by $\tau^{\prime}$. Therefore the covering $\pi: S /\langle\tau\rangle \rightarrow S^{\prime} /\left\langle\tau^{\prime}\right\rangle$ ramifies over the boundary. This discards the signature $\sigma_{2}$ and we deduce, without any other computation, that $\pi$ does not ramify over the interior and it ramifies exactly over four points in the boundary, whose branching orders are 2,2, $m$ and $m$.

We can explicitly compute these branch points. Repeating the argument above it follows that $p$ and $p^{\prime}=\pi(1,-\rho)$ are the two branch points of $\pi$ with branching order 2. As to the two points with branching order $m$, they must be projections under $\pi$ of points in $S$ fixed by $u:(z, w) \mapsto\left(\xi_{m} z, w\right)$. Using the above formula of $\pi$, it follows easily that the two branch points of branching order $m$ of $\pi$ are the points at infinity of $S^{\prime}$.

Cyclic and dihedral $n$-sheeted coverings with $n>2$ have been treated separately in Theorems 4.3 and 4.5, respectively. As we pointed out, for $n=2$ the groups $C_{n}$ and $D_{n / 2}$ coincide, and this is the reason why the result for double coverings requires a separate statement. The content of Theorem 4.9 has been split into two parts: part (i) corresponds to Theorem 4.3 and part (ii) corresponds to Theorem 4.5 . 
4.9. Theorem. Let $\pi:(S, \tau) \rightarrow\left(S^{\prime}, \tau^{\prime}\right)$ be a branched, double covering where $(S, \tau)$ is a hyperelliptic real algebraic $M$-curve of algebraic genus $g \geq 2$. Let $u$ be the involution on $(S, \tau)$ such that $S^{\prime}=S /\langle u\rangle$. Assume that $u$ is not the hyperelliptic involution of $S$. Then, up to dianalytic equivalence, the covering $\pi:(S, \tau) \rightarrow\left(S^{\prime}, \tau^{\prime}\right)$ can be represented in terms of algebraic equations in one of the following forms:

(i)

$$
S: w^{2}=\prod_{j=1}^{g+1}\left(z^{2}-a_{j}^{2}\right) \quad \text { and } \quad \tau(z, w)=\left(\frac{1}{\bar{z}}, \frac{\bar{w}}{\bar{z}^{g+1}}\right),
$$

with $\left|a_{j}\right|=1$ for all $j, a_{j}^{2} \neq a_{i}^{2}$ for $i \neq j$ and $\prod_{j=1}^{g+1} a_{j}^{2}=1$. The covering transformation group $G$ is generated by the automorphism

$$
u_{\varepsilon}:(z, w) \rightarrow\left(-z,(-1)^{\varepsilon} w\right) \text {, where either } \varepsilon=0 \text { or } \varepsilon=1 .
$$

For each value of $\varepsilon$ the covered curve is $\left(S^{\prime}, \tau^{\prime}\right)$ where

$$
S^{\prime}: W^{2}=Z^{\varepsilon} \prod_{j=1}^{g+1}\left(Z-a_{j}^{2}\right), \quad \tau^{\prime}(Z, W)=\left(\frac{1}{\bar{Z}}, \frac{\bar{W}}{\bar{Z}^{\varepsilon+(g+1) / 2}}\right),
$$

and the covering projection is given by

$$
\pi:(z, w) \mapsto(Z, W)=\left(z^{2}, z^{\varepsilon} w\right) .
$$

(ii) There exist $\varepsilon_{1}, \varepsilon_{2} \in\{-1,+1\}$ such that:

$$
S: w^{2}=\prod_{j=1}^{g+1}\left(z-a_{j}\right)\left(z-\bar{a}_{j}\right) \quad \text { and } \quad \tau(z, w)=\left(\frac{1}{\bar{z}}, \frac{\varepsilon_{1} \bar{w}}{\bar{z}^{g+1}}\right),
$$

with $\left|a_{j}\right|=1$ for all $j$ and such that the polynomial in the variable $z$ on the right-hand side has no multiple roots. The covering transformation group is generated by

$$
u:(z, w) \rightarrow\left(\frac{1}{z}, \frac{\varepsilon_{2} w}{z^{g+1}}\right) .
$$

Let $b_{j}=a_{j}+\bar{a}_{j}$ for all $j$. Then the curve $\left(S^{\prime}, \tau^{\prime}\right)$ and the projection $\pi$ are given by

$$
S^{\prime}: W^{2}=(Z-2)^{\varepsilon_{3}}(Z+2)^{\varepsilon_{4}} \prod_{j=1}^{g+1}\left(Z-b_{j}\right), \quad \tau^{\prime}(Z, W)=\left(\bar{Z}, \varepsilon_{1} \varepsilon_{2} \bar{W}\right),
$$

and

$$
\pi(z, w)=(Z, W)=\left(z+\frac{1}{z}, \frac{w(z-1)^{\varepsilon_{3}}(z+1)^{\varepsilon_{4}}}{z^{\left(\varepsilon_{3}+\varepsilon_{4}+g+1\right) / 2}}\right),
$$

where $\varepsilon_{3}=\left(1-\varepsilon_{2}\right) / 2$ and $\varepsilon_{4}=\left(1+(-1)^{g} \varepsilon_{2}\right) / 2$.

The proof of this theorem is omitted since it follows from those of Theorems 4.3 and 4.5. see also [5]. However, some comments are in order. Unlike $n$-sheeted coverings with $n>2$, it is more convenient now to begin by choosing the antianalytic involution $\widehat{\tau}$ induced by $\tau$ as $\widehat{\tau}: z \mapsto 1 / \bar{z}$, and finding then the analytic involution $\widehat{u}$ commuting with $\widehat{\tau}$. If $n>2$, then $\widehat{u}: z \mapsto \xi_{n} z$ is unique with this property (up to conjugation which preserves $\widehat{\tau}$ ). However, if $n=2$, then $\widehat{u}: z \mapsto 1 / z$ also commutes with $\widehat{\tau}$. In fact these two are the only involutions $\widehat{u}$ commuting with $\widehat{\tau}$ up to conjugation which preserves $\widehat{\tau}$. The first one corresponds to part (i) in the theorem whilst the second one corresponds to part (ii). Once this is achieved, the 
proof runs parallel to those in Theorems 4.3 and 4.5 . Finally note that the coverings in part (i) just occur if the genus $g$ is odd.

The next comment just involves normal coverings between hyperelliptic complex curves, independently of their symmetric character and dihedral or cyclic actions.

4.10. Remark. It follows from Lemma 4.1 that the set $W\left(S^{\prime}\right)$ of Weierstrass points of the algebraic curve $S^{\prime}$ contains the projection $\pi(W(S))$ of the set of Weierstrass points of $S$. We wonder whether $\pi(W(S))$ is strictly contained in $W\left(S^{\prime}\right)$.

It is easy to prove that for each automorphism $\gamma \in G$,

$$
\operatorname{Fix}(\gamma \circ h) \subseteq \operatorname{Fix}(h) \Longleftrightarrow \pi(\operatorname{Fix}(\gamma \circ h)) \subseteq \pi(\operatorname{Fix}(h)),
$$

where $h$ is the hyperelliptic involution of $S$. Thus we deduce from Lemma 4.1 that

$$
\pi(W(S))=W\left(S^{\prime}\right) \Longleftrightarrow \operatorname{Fix}(\gamma \circ h) \subseteq W(S) \text { for all } \gamma \in G .
$$

For the coverings we are dealing with, the algebraic presentations given in Theorems 4.3 and 4.5 yield straightforwardly that in the cyclic case $W\left(S^{\prime}\right)=\pi(W(S))$ if and only if $\varepsilon=0$ (otherwise both sets differ in just one point), whilst in the dihedral case $W\left(S^{\prime}\right)=\pi(W(S))$ if and only if $\varepsilon_{4}=\varepsilon_{5}=0$ (otherwise both sets differ in $\varepsilon_{4}+\varepsilon_{5}$ points).

Moreover, it also follows from the given algebraic presentation that the set $\pi(W(S))$ contains no branch point of the covering $\pi: S \rightarrow S^{\prime}$. In fact, the same holds true for the whole set $W\left(S^{\prime}\right)$ unless the covering is cyclic and $\varepsilon=1$.

4.11. Remark. We are assuming throughout the paper that $G$ does not contain the hyperelliptic involution. Otherwise, the quotient $S^{\prime}=S / G$ would be the Riemann sphere. However, it is possible for the action of $G$ to yield a genus zero quotient (and even genus one) with $G$ not containing the hyperelliptic involution. This can be checked using the Riemann-Hurwitz formula. In fact, our description of coverings by means of algebraic equations allows us to find all the cases in which this phenomenon occurs. Recall that the genus of the curve $S^{\prime}$ represented as $w^{2}=P(z)$ is $[(\operatorname{deg} P-1) / 2]$, where [.] stands for the integer part.

Suppose first that $G$ is a cyclic group. Then $n$ divides $g+1$ and $\operatorname{deg} P=$ $\varepsilon+(2 g+2) / n$ where $\varepsilon=0$ or 1 . Hence $S^{\prime}$ has genus zero if and only if $\operatorname{deg} P=2$, i.e., $\varepsilon=0$ and $g+1=n$. Since $\tau^{\prime}$ fixes points in $S^{\prime}$, the Klein surface $S^{\prime} /\left\langle\tau^{\prime}\right\rangle$ is a closed disk. Similarly, $S^{\prime}$ has genus one if and only if either $\varepsilon=1$ and $g+1=n$, or $\varepsilon=0$ and $g+1=2 n$. Counting the number of branch points of $S^{\prime}$ fixed by $\widehat{\tau}^{\prime}$, it follows from [2, Thm. 17.20] that $S^{\prime} /\left\langle\tau^{\prime}\right\rangle$ is a Möbius band in the first case and a closed annulus in the second.

Suppose now that $G$ is a dihedral group. Then, $S^{\prime}$ is represented as $w^{2}=P(z)$ where $\operatorname{deg} P=\varepsilon_{4}+\varepsilon_{5}+(g+1) / m$ with $\varepsilon_{4}, \varepsilon_{5}=0$ or 1 . Hence $S^{\prime}$ has genus zero if and only if either $\varepsilon_{4}=\varepsilon_{5}=0$ and $(g+1) / m=1$ or 2 , or $\varepsilon_{4}+\varepsilon_{5}=(g+1) / m=1$. Again, the Klein surface $S^{\prime} /\left\langle\tau^{\prime}\right\rangle$ is a closed disk. As to the elliptic case, $S^{\prime}$ has genus one if and only if one of the following three possibilities occur: (i) $\varepsilon_{4}=\varepsilon_{5}=0$ and $(g+1) / m=3$ or $4 ; \quad$ (ii) $\varepsilon_{4}+\varepsilon_{5}=1$ and $(g+1) / m=2$ or $3 ; \quad$ (iii) $\varepsilon_{4}=\varepsilon_{5}=1$ and $(g+1) / m=1$ or 2 . In any case the symmetry $\widehat{\tau}^{\prime}$ fixes all of the branch points of $S^{\prime}$, and so $S^{\prime} /\left\langle\tau^{\prime}\right\rangle$ is a closed annulus.

\section{ACKNOWLEDGMENT}

We would like to thank Professor Warren Dicks for his valuable comments on a previous version which improved the presentation of the paper. 


\section{REFERENCES}

[1] R. D. M. Accola, On lifting the hyperelliptic involution, Proc. Amer. Math. Soc., 122 (2), (1994), 341-347. MR1197530 (95a:14029)

[2] N. L. Alling, Real Elliptic Curves, Mathematical Studies 54, North-Holland, 1981. MR640091 (84d:14027)

[3] N. L. Alling and N. Greenleaf, Foundations of the Theory of Klein Surfaces, Lecture Notes in Math. 219, Springer, 1971. MR0333163 (48:11488)

[4] E. Bujalance, A classification of unbranched double coverings of hyperelliptic Riemann surfaces, Arch. Math. 47, (1) (1986), 93-96. MR855143 (87k:14030)

[5] E. Bujalance, F. J. Cirre, J. M. Gamboa, Double coverings of hyperelliptic real algebraic curves, submitted.

[6] E. Bujalance, J. J. Etayo, J. M. Gamboa, Hyperelliptic Klein surfaces, Quart. J. Math. Oxford, 36 (2) (1985), 141-157. MR790476 (86g:30057)

[7] E. Bujalance, J. J. Etayo, J. M. Gamboa, G. Gromadzki, Automorphisms Groups of Compact Bordered Klein Surfaces, Lecture Notes in Math. 1439, Springer-Verlag, Berlin, Heidelberg, 1990. MR1075411 (92a:14018)

[8] F. J. Cirre, Birational classification of hyperelliptic real algebraic curves, The Geometry of Riemann Surfaces and Abelian Varieties, Contemporary Math. 397 (2006), 15-26. MR2217994 (2007a:14034)

[9] H. M. Farkas, Unramified double coverings of hyperelliptic surfaces, J. Analyse Math. 20 (1976), 150-155. MR0437741 (55:10664)

[10] H. M. Farkas, Unramified double coverings of hyperelliptic surfaces II, Proc. Amer. Math. Soc., 101 (3) (1987), 470-474. MR.908651 (88k:30052)

[11] Y. Fuertes, G. González-Diez, Smooth double coverings of hyperelliptic curves. The Geometry of Riemann Surfaces and Abelian Varieties, Contemporary Math., 397 (2006), 73-77, MR.2217998 (2006m:14042)

[12] Y. Fuertes, G. González-Diez, On unramified normal coverings of hyperelliptic curves. J. of Pure and Applied Algebra, 208 (3) (2007), 1063-1070. MR2283446

[13] B. H. Gross, J. Harris, Real algebraic curves, Ann. Sci. École Norm. Sup., 14 (1981), 157-182. MR631748 (83a:14028)

[14] A. Harnack, Uber die Vieltheiligkeit der ebenen algebraischen Kurven, Mat. Ann., 10 (1876), 189-198. MR.1509883

[15] R. Horiuchi, Normal coverings of hyperelliptic Riemann surfaces, J. Math. Kyoto Univ. 19 (3) (1979), 497-523. MR553229 (80k:30044)

[16] E. Kani, Unramified double covers of hyperelliptic Klein surfaces, C. R. Math. Rep. Acad. Sci. Canada 9 (3) (1987), 133-138. MR888645 (88f:30079)

[17] C. Maclachlan, Smooth coverings of hyperelliptic surfaces, Quart. J. Math. Oxford (2) 22 (1971), 117-123. MR0283194 (44:427)

[18] H. H. Martens, A remark on Abel's Theorem and the mapping of linear series, Comment. Math. Helvetici 52 (1977), 557-559. MR0460325 (57:319)

Departamento de Matemáticas Fundamentales, Facultad de Ciencias, Universidad Nacional de Educación a Distancia, Madrid, Spain

E-mail address: ebujalance@mat.uned.es

Departamento de Matemáticas Fundamentales, Facultad de Ciencias, Universidad Nacional de Educación a Distancia, Madrid, Spain

E-mail address: jcirre@mat.uned.es

Departamento de Álgebra, Facultad de Ciencias Matemáticas, Universidad ComPLUTEnse de MAdrid, Madrid, Spain

E-mail address: jmgamboa@mat.ucm.es 